\title{
Multiscale Cardiac Imaging: From Whole Heart Images to Cardiac Ultrastructure
}

Graham Rykiel ${ }^{1}$, Claudia S. López ${ }^{1,3}$, Jessica L. Riesterer ${ }^{1,3}$, Melissa Williams ${ }^{1,3}$, Katherine Courchaine $^{1}$, Alina Maloyan ${ }^{2}$, Kent Thornburg ${ }^{2}$ and Sandra Rugonyi ${ }^{1,2^{*}}$

1. Biomedical Engineering, Oregon Health \& Science University, Portland, Oregon, USA.

2. Center for Developmental Health, Knight Cardiovascular Institute, Oregon Health \& Science University, Portland, Oregon, USA.

3. Multiscale Microscopy Core, Oregon Health \& Science University, Portland, Oregon, USA.

*Corresponding author: rugonyis@ohsu.edu

The heart is a multiscale biological pump. Structurally, the heart has four chambers, and cardiac walls are formed by myocardial cells, which are elongated cells that actively contract along their axial direction. At the sub-cellular or ultrastructural level, cardiomyocytes contain myofibrils, the heart's contractile units, aligned along the cells' long axis and surrounded by tightly arranged energy-supplying mitochondria [1]. Changes in the organization or quantity of contractile elements impact cardiac function. In cases of congenital heart defects, moreover, this contractile machinery is affected by both the structural heart malformation and ultrastructural anomalies. To understand functional deficits associated with congenital heart defects, therefore, it is important to study both the morphological malformation and associated ultrastructural changes. This requires a multiscale imaging approach that allows mapping of ultrastructure to the whole heart morphology. While structural images of the whole heart can be acquired with X-ray micro-computed tomography (microCT) at micron resolution; ultrastructural images are acquired with electron microscopy (EM) at nanometer resolution. These two imaging modalities can be mutually exclusive, given the disparate size of samples and preparation protocols. To circumvent this limitation, we have developed a multiscale imaging pipeline that allows acquisition of microCT images of the whole heart, followed by selection of specific regions of interest (ROIs) and acquisition of 3D serial block face scanning EM (SBF-SEM) images from the same heart.

Our multiscale imaging approach consists of four steps (see Figure 1A). In step 1, the heart was prepared for microCT imaging using a protocol compatible with later SBF-SEM imaging. To this end, we fixed the whole heart uniformly, by perfusion and immersion in fixative (2.5\% Glutaraldehyde and 2.5\% Paraformaldehyde). Next, the heart was incubated in solutions containing Osmium Tetroxide, Potassium Ferricyanide and Thiocarbohydrazide for staining, after which the heart was imaged with microCT (Caliper Quantum FX; $10 \mu \mathrm{m}$ resolution). In step 2, the heart was incubated in uranyl acetate and lead aspartate solutions and embedded into a resin (Epon) block to complete tissue preparation for SBF-SEM. Using the microCT images as reference for ROI positioning, the resin block was sectioned, and a semithin section $(250 \mathrm{~nm})$ obtained to confirm the ROI selection and check ultrastructural quality. In Step 3, a slab ( $\sim 1.5 \mathrm{~mm}$ thick) of sample was cut and sectioned to generate a pillar of 500x500 $\mu \mathrm{m}^{2}$ from which ROIs of $40 \times 60 \mu \mathrm{m}^{2}$ were selected. In Step 4, 3D SBF-SEM datasets were acquired (Teneo VolumeScope; $10 \mathrm{~nm}$ lateral resolution). Our approach allows imaging of the whole heart structure and its sub-cellular architecture, as well as mapping of SEM images to microCT images.

We employed our pipeline to image the hearts of our chicken models of congenital heart defects [2]. It is worth mentioning, however, that extension to other small animal models (e.g. mouse) should be straightforward. In our study, 3D microCT cardiac images were used to identify two hearts (see Figure 1B): one sham control heart with no structural defects, and one heart with tetralogy of Fallot (TOF). The 
selected hearts were fully processed, and we obtained 3D SBF-SEM images from both hearts at corresponding locations within the left ventricular wall. Analysis of 3D SBF-SEM data sets showed excellent ultrastructure preservation with intact nuclear envelopes and consistent staining (see Figure 2). Ultrastructural analysis revealed regions of highly aligned myofibrils with mitochondria packed along them in the control heart, whereas the TOF heart presented regions of disarrayed myofibrils surrounded by sparse mitochondria. Thus ultrastructural characteristics of the TOF heart likely compromise cardiac performance beyond the structural defects. By combining whole heart microCT imaging with 3D SBFSEM of cardiac ROIs, our novel imaging pipeline enables multiscale studies of cardiac function [3].

\section{References:}

[1] C Pinali and A Kitmitto, Journal of Molecular and Cellular Cardiology 76 (2014), p. 1.

[2] M Midgett, KL Thornburg and S Rugonyi, American Journal of Physiology - Heart and Circulatory Physiology 312 (2017), p. H632.

[3] The authors would like to acknowledge funding from NIH R01 HL094570; the OHSU University Shared Resource pilot funding program; and the OHSU School of Medicine Faculty Innovation Fund.

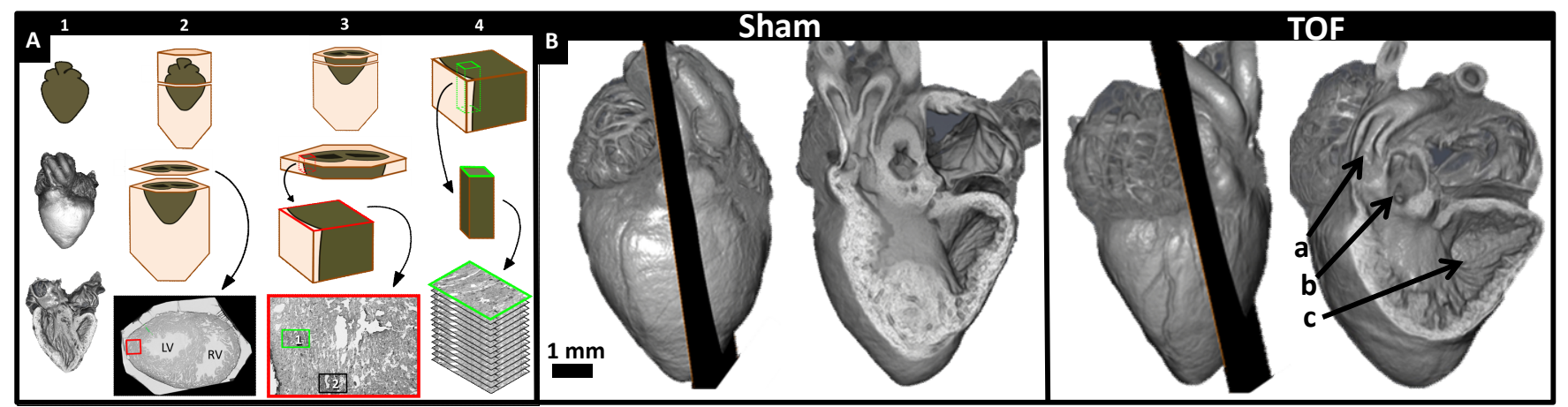

Figure 1. Multiscale imaging of the heart. (A) Multiscale imaging pipeline. Our 4-step approach yielded whole heart and ultrastructural images from the same heart. Step 1 ends with microCT images of the whole heart. In Step 2 contrast staining is finalized and the resin block in which the heart is embedded is sectioned to reach a plane of interest. In step 3, a sample slab is cut and mounted. In Step 4, the selected region of interest is imaged using 3D SBF-SEM to reveal ultrastructural features. (B) MicroCT images of the whole heart from which cardiac defects can be identified. A cut plane is also shown. Normal heart (sham) vs heart with tetraology of Fallot (TOF) malformation characterized by a) pulmonary artery stenosis; b) conoventricular septal defect; c) right ventricular hypertrophy; and over-riding aorta (not shown). Scale bar $=1 \mathrm{~mm}$.

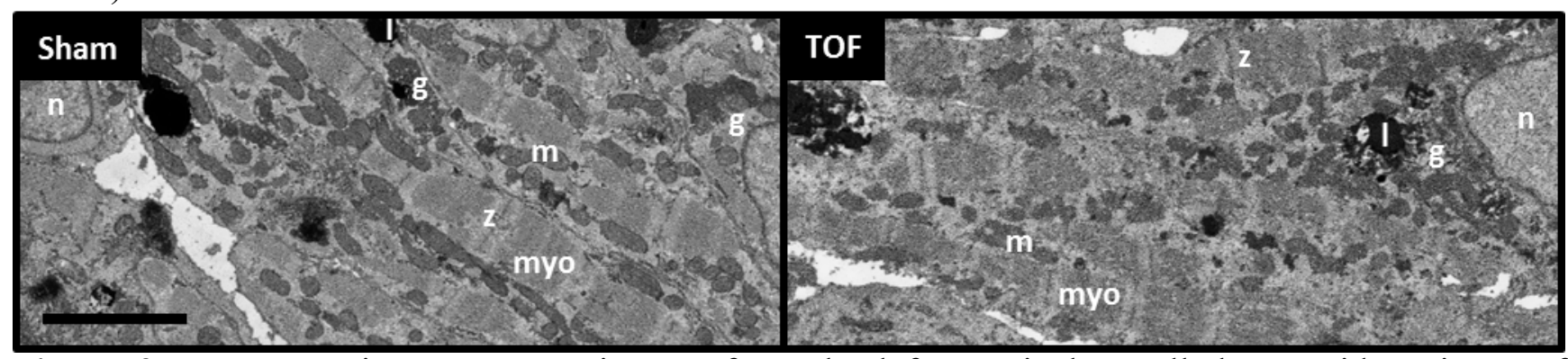

Figure 2. Representative SBF-SEM images from the left ventricular wall, heart mid-section. Left: normal (sham) heart ultrastructure. Right: TOF heart ultrastructure. Labels correspond to: myofibrils (myo); z-disks (z); mitochondria (m); nucleus (n); glycogen (g); lipid droplet (l). Scale bar $=3 \mu \mathrm{m}$. 